\title{
Getting involved in ENT clinical research in the UK; how can the NIHR Clinical Research Network help?
}

\author{
Blackshaw, H., * Philpott, C., ${ }^{\dagger}$ Bhutta, M.F., * Cooper, S. ${ }^{\ddagger}$ \& Schilder, A.G.M.* \\ ${ }^{*}$ evidENT, Ear Institute, University College London, London, UK, ${ }^{\dagger}$ Norwich Medical School, University of East Anglia, Norwich, UK, \\ ${ }^{\ddagger}$ NIHR Clinical Research Network Coordinating Centre, Leeds, UK
}

Clin. Otolaryngol. 2014, 39, 328-333

\section{Introduction to the National Institute for Health Research}

In 2006, the Department of Health formed the National Institute of Health Research (NIHR) to create an environment for world class health research within the National Health Service (NHS). Historically, research occurred mainly in teaching hospitals, with a lack of equal opportunity for patients to participate in research and no overall nationwide framework to assist the conduct of research within the NHS. With the mission to 'Improve the health and wealth of the nation through research', the NIHR has transformed clinical research in the United Kingdom through the establishment of the hugely successful Clinical Research Network (CRN) and the initiation of a national portfolio of studies, together providing the local infrastructure to support clinical researchers in conducting highquality research. This has led to an increased number of healthcare professionals and patients participating in research and the NHS being seen as an international leader in clinical research.

\section{What is the NIHR?}

- The National Institute for Health Research is a national organisation operating within the NHS with the mission to improve the health and wealth of the nation through research

- It is led by the Chief Medical Officer Professor Dame Sally Davis

- The overall annual budget is ring-fenced at $1 \%$ of the NHS budget ( $£ 1$ billion)

Academics, clinicians, industries and patients involved in ENT clinical research may already be aware of the CRN and the support they offer for the delivery of research. Equally, the CRN may be viewed by some as a familiar name, but a vast and complex organisation of unknown capacity. Or possibly, the CRN is a completely unknown entity. This brief paper aimed to provide practical information to enable ENT, hearing and balance specialties to benefit from the research infrastructural support provided by the CRN. We will focus on the impact the network has had on recent ENT studies as well as an introduction to the recognisable names behind the network and the support it offers.

\section{What is the Clinical Research Network?}

- The CRN was set up by the NIHR to enable NHS patients and health professionals across England to participate in high-quality relevant clinical research

- The annual budget for the CRN is $£ 286 \mathrm{~m}$

- The CRN provides a unique health service infrastructure to support clinical research in the NHS

\section{Where does ENT fit in the structure of the NIHR Clinical} Research Network?

The CRN comprises of 15 Local Clinical Research Networks (LCRNs) that cover the length and breadth of England (Fig. 1). Each LCRN delivers research across 30 clinical specialties including ENT. A national coordinating centre provides central management for the network. Similar system exists within the devolved nations and details regarding those can be found at: http://www.cso.scot.nhs. uk/, http://www.nicrn.hscni.net/ and http://www.wales.nhs. $\mathrm{uk} / \mathrm{sites} 3 /$ home.cfm? orgid $=952$.

\section{The NIHR CRN Portfolio, what is it and what are the benefits?}

At the heart of the CRN's activities is the NIHR CRN Portfolio. This consists of high-quality clinical research studies that are eligible for consideration for support from the CRN in England.

The portfolio database captures research activity data and provides analysis tools to facilitate active management of current studies, and the feasibility of future studies run within the CRN. Activity data from the portfolio are used to 

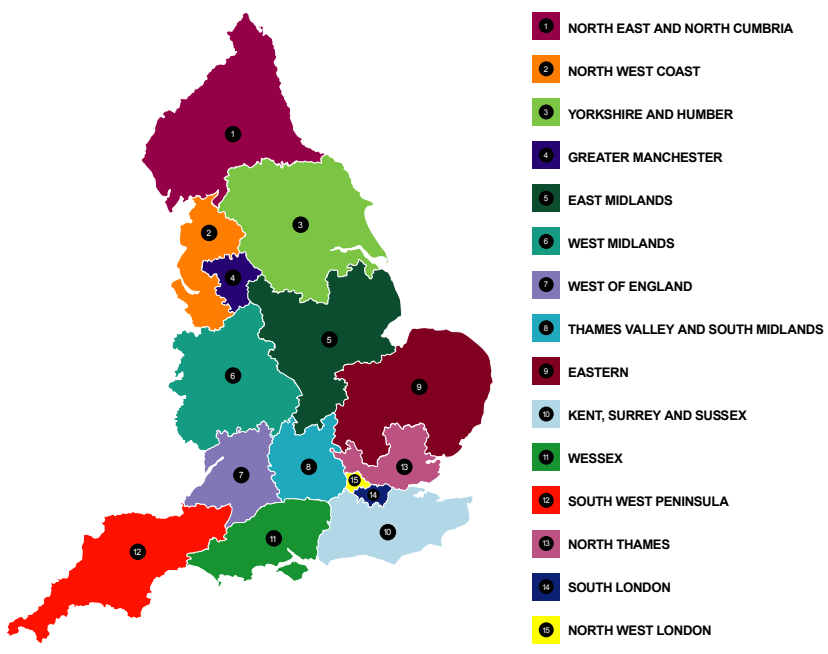

Fig. 1. NIHR Local Clinical Research Networks in England.

inform the allocation of NHS infrastructure for research (including NHS support costs) and support the performance management of the CRN.

There have been over 90 observational and interventional Ear, Nose and Throat studies registered on the portfolio as it was set up and over 30 of these are currently recruiting participants within the NHS (http://public.ukcrn.org.uk/ search/). Clinical studies registered on the portfolio benefit from the CRN infrastructure that has been put in place to assist researchers to complete their research, including the following:

- Access to a local network of dedicated skilled research support staff including research nurses and other allied health professionals, who can help identify eligible patients, arrange consent to participate in the study and monitor patients as they progress through the study

- Support to ensure that a given study can be successfully undertaken in the NHS including pharmacy, imaging and pathology services and the possibility of securing protected time for NHS staff to conduct research

- Provision of dedicated systems to assist researchers in gaining approvals to open their studies within the NHS quickly and efficiently (Coordinated System of Permissions, CSP). In the past, researchers had to make separate permission applications to every trust, but the CRN has revolutionised this process through CSP. Researchers now only enter their study details once, even on multisite studies. The CRN then coordinates all the necessary NHS Research and Development reviews at every participating NHS Trust through CSP.

- Practical help in identifying and recruiting patients onto portfolio studies, so that researchers can be confident of completing the study on time and with the target number of participants required.

- National monitoring of the monthly performance of portfolio studies and the availability of local dedicated ENT, hearing and balance experts to assist with specialist knowledge to help recruit participants to studies (Table 1).

All researchers with funded ENT research projects should therefore consider applying for portfolio inclusion to gain advantage of this support the CRN offers and bring this additional funding for research delivery into their trust.

\section{How can i get my ENT study adopted onto the portfolio?}

Both non-commercial (researcher-led) and commercial (industry-led) studies can register to be included in the portfolio. As the CRN is a government funded organisation, all studies in England must comply with the Department of Health established eligibility criteria for commercial and non-commercial research to qualify for CRN support.

Table 1. Who is my ENT specialty local lead? Who can I contact for specialist advice?

\begin{tabular}{lll} 
Local clinical research network (LCRN) & ENT specialty local lead & Specialism \\
East Midlands & Professor Deborah Hall & Audiology \\
Eastern & Mr Carl Philpott & Rhinology, Anterior skull base \\
Greater Manchester & Mr Kevin Green & Otology \\
North East and North Cumbria & Mr Anirvan Banerjee & Otology \\
North Thames & Professor Anne Schilder & Paediatric ENT, Clinical trials methodology \\
North West Coast & Mr Adam Donne & Paediatric ENT \\
North West London & Mr Taran Tatla & Laryngology, Head and neck \\
Scotland & Mr Kenneth MacKenzie & Laryngology \\
South London & Ms Claire Hopkins & Rhinology, Anterior skull base \\
Thames Valley and South Midlands & Mr James Ramsden & Otology, Paediatric ENT \\
Yorkshire and Humber & Mr Jaydip Ray & Otology, Paediatric ENT \\
\hline
\end{tabular}




\section{Non-commercial studies}

Eligibility for inclusion is judged by experts within the CRN to ensure the research has been funded in open national competition, appropriately peer-reviewed, is of clear value to the NHS and fits within the needs and realities of the NHS.

Researchers completing their approval forms within the Integrated Research System (IRAS) can choose on the first page of their IRAS application to have their project assessed for CRN support and inclusion in the CRN Portfolio. If you choose to be considered for portfolio inclusion, you will be asked to complete and submit a CRN portfolio application form (PAF). Only one PAF is required for the entire research project from the lead site, where the Chief Investigator is based. Within 2 working days, you will receive confirmation that you can use the Coordinated System for Gaining NHS Permission (CSP) system through which you should complete and submit your Research and Development (R\&D) application. The eligibility process runs in parallel to the R\&D checks and a decision on eligibly for the NIHR Portfolio will then be given within 30 days. In 2013/14, the median number of days to achieve NHS Permission for all study sites for all portfolio studies was $\mathbf{2 5}$ calendar days (http://www.crn.nihr.ac.uk/about-crn/our-performance/key-statistics-2/).

Further information on the process can be found at: http://www.crn.nihr.ac.uk/can-help/funders-academics/ gaining-nhs-permissions/the-csp-flowchart/).

\section{Commercial studies}

The CRN provides free support to help the life sciences industry to deliver high-quality research in the NHS. The CRN's priority is to proactively support the life sciences industry to deliver high-quality clinical research in the NHS, and to enable this, they have developed a range of free services and support tools around feasibility, set-up and performance management.

To be eligible for inclusion on the portfolio, commercial studies (i.e. those which are industry-led and either fully or partly industry supported) must be submitted to the network via an online system by a member of the sponsoring company or Clinical Research Organisation (CRO) for the assessment against the following eligibility criteria, as defined by the Department of Health:

- The study must meet the networks definition of 'research' (further information can be found at the web link below).

- The study must receive NHS Research Ethics Committee approval and NHS permission prior to initiation at individual sites.
Researchers wishing to introduce commercial companies to the network should direct them to the website http://www. crn.nihr.ac.uk/can-help/life-sciences-industry/. Companies can submit their studies to the network at www.submitmystudy.nihr.ac.uk.

\section{Success story 1}

Established in October 2007, the Chronic Rhinosinusitis Epidemiology Study (CRES) was run in ENT departments in East Anglia. The study set out to recruit 1000 patients with chronic rhinosinusitis and 200 control subjects. The aim was to investigate the medical, socio-economic, psychological and environmental factors that may influence the severity of chronic rhinosinusitis and so address one of the research objectives of the European Position Paper on Rhinosinusitis and Nasal Polyps. ${ }^{1}$

From 2007 to 2012, a total of 627 participants were recruited at a rate of about 7 per month. Following appointment at the University of East Anglia as a Senior Lecturer, Mr Carl Philpott (Eastern ENT local lead) used funding from the Anthony Long Trust to establish this as a study eligible for inclusion on the portfolio.

Adoption onto the portfolio in August 2012, together with the support of the national ENT specialty, raised the profile of this study enabling it to be expanded to numerous sites throughout the United Kingdom (Fig. 2). The geographic distribution of study participants included a wide range of rural and urban areas of England and 2 of 3 devolved nations (Fig. 3). CRN support included provision of funding for research nurses or administrative input with the greatest network input seen in the North East, resulting in recruit-

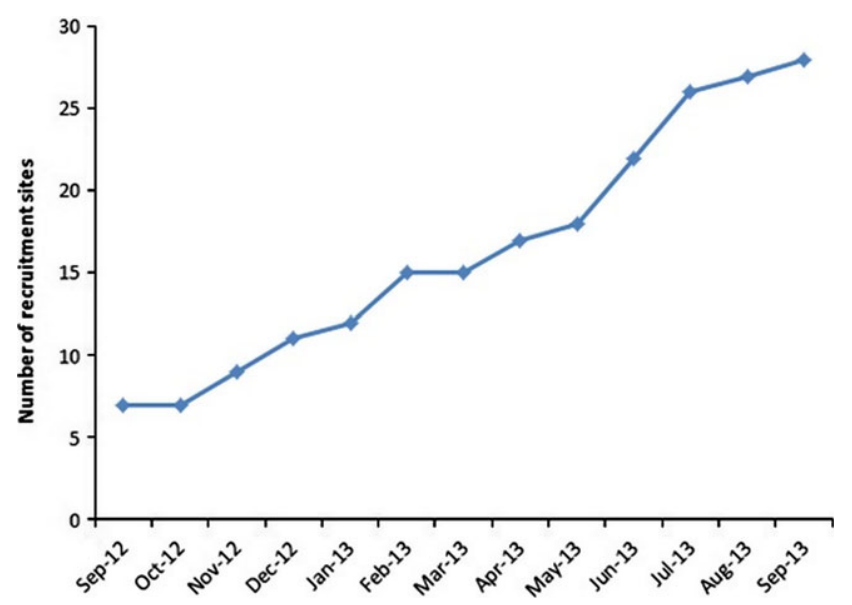

Fig. 2. Number of open recruitment sites in the Chronic Rhinosinusitis Epidemiology Study (CRES) following portfolio inclusion in August 2012. 


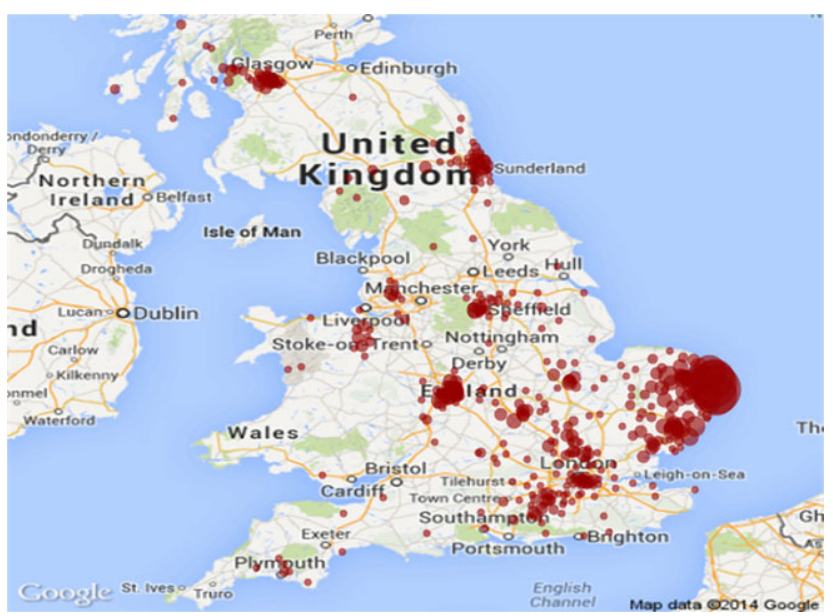

Fig. 3. Geographical spread of recruitment sites for the Chronic Rhinosinusitis Epidemiology Study (CRES).

ment of the highest number of participants outside of East Anglia. Overall, the number of study participants entering the study each month rose from the seven recruited in September 2012 to a peak of 130 participants in August 2013 (Fig. 4).

With this successful UK-wide support, the study completed recruitment of 1552 participants in September 2013, with 1480 complete data sets recorded in the final database. Study data are currently being analysed at the University of East Anglia and once published will provide further impetus for research initiatives in rhinosinusitis in the United Kingdom.

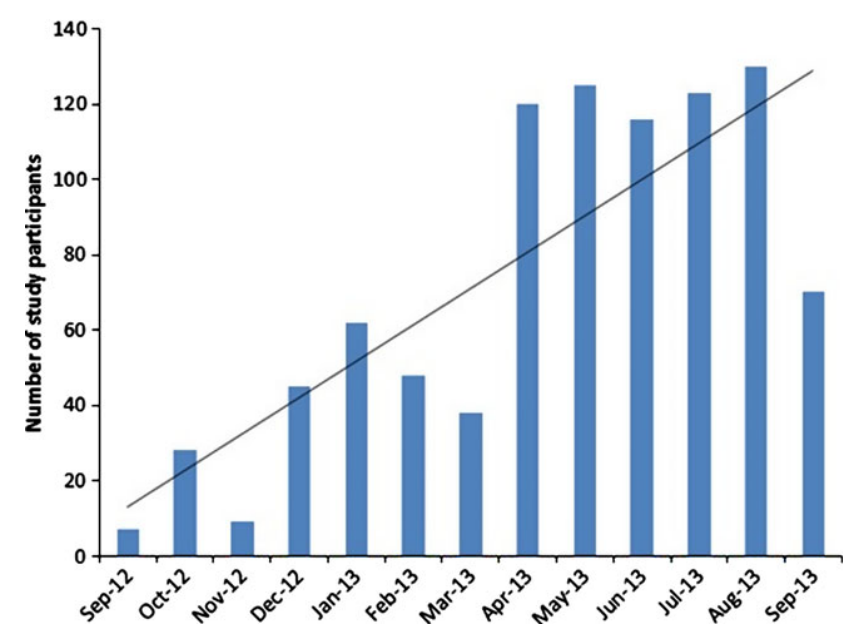

Fig. 4. Number of patients recruited to the Chronic Rhinosinusitis Epidemiology Study (CRES) per month following portfolio inclusion in August 2012.

\section{What is the NIHR ENT specialty?}

'Specialties' were set up by the NIHR CRN to provide national networks of topic-specific expertise. The aim of the ENT specialty is to ensure the successful completion of NIHR Portfolio research studies within ENT, hearing and balance. Each specialty is formed of clinical researchers who represent their local network and act as local leads. Each local lead is able to provide guidance on the development and conduct of research within their region. In addition, these local members form a national network of expertise across all areas of healthcare.

The ENT specialty currently has 11 local leads spanning the United Kingdom with a wide range of subspecialty expertise (Table 1). The member currently appointed as national lead for the ENT specialty is Professor Anne Schilder.

Local leads, together with additional members representing the devolved nations and important ENT stakeholders, meet three times a year to discuss national ENT research and the support they can provide to portfolio studies. For example, they identify the portfolio studies that are struggling to recruit participants and those that need to open up to additional sites. In addition, the specialty is able to provide detailed subspecialty clinical advice to researchers investigating a specific condition/area within ENT. Local leads are able to provide details of patient populations within their region, so promoting study opening within new NHS Trusts and enhancing the number of patients offered the opportunity to participate in research across the United Kingdom.

The ENT specialty works to engage the wider ENT community in the work of the CRN to raise awareness of the support the network provides. As a result, a coordinated national message is being spread to researchers, ENT research initiatives are starting to broaden from local studies to multicentre trials, and the proportion of open studies on the portfolio recruiting the required number of participants within the timescale of the funding has improved.

The ENT specialty has also organised successful workshop sessions, open to the wider ENT community, with themes such as Patient and Public Involvement (2012, 2014), Working with Industry (2013) and Developing Research Questions and Prioritisation in collaboration with NETSCC 2013).

\section{Success story 2}

The chief investigator of the Genetics of Otitis Media study, Mr Mahmood Bhutta, believes the success of this study is down to the network support he and his team 


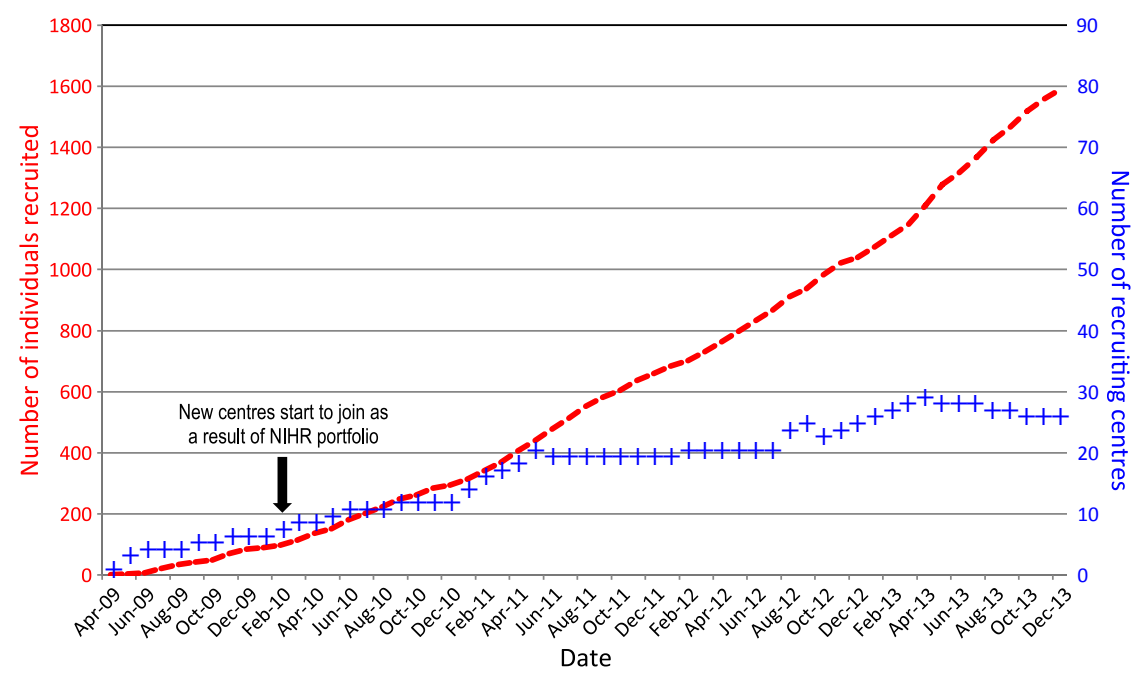

Fig. 5. Recruitment to the genetics of otitis media study April 2009 to December 2013.

received. Otitis media is known to be highly heritable, and his study set out to discover candidate genetic polymorphisms conferring susceptibility, through recruitment of children having grommets inserted to treat chronic glue ear.

Power calculations showed that at least 900 affected families needed to be recruited to discover genes of moderate

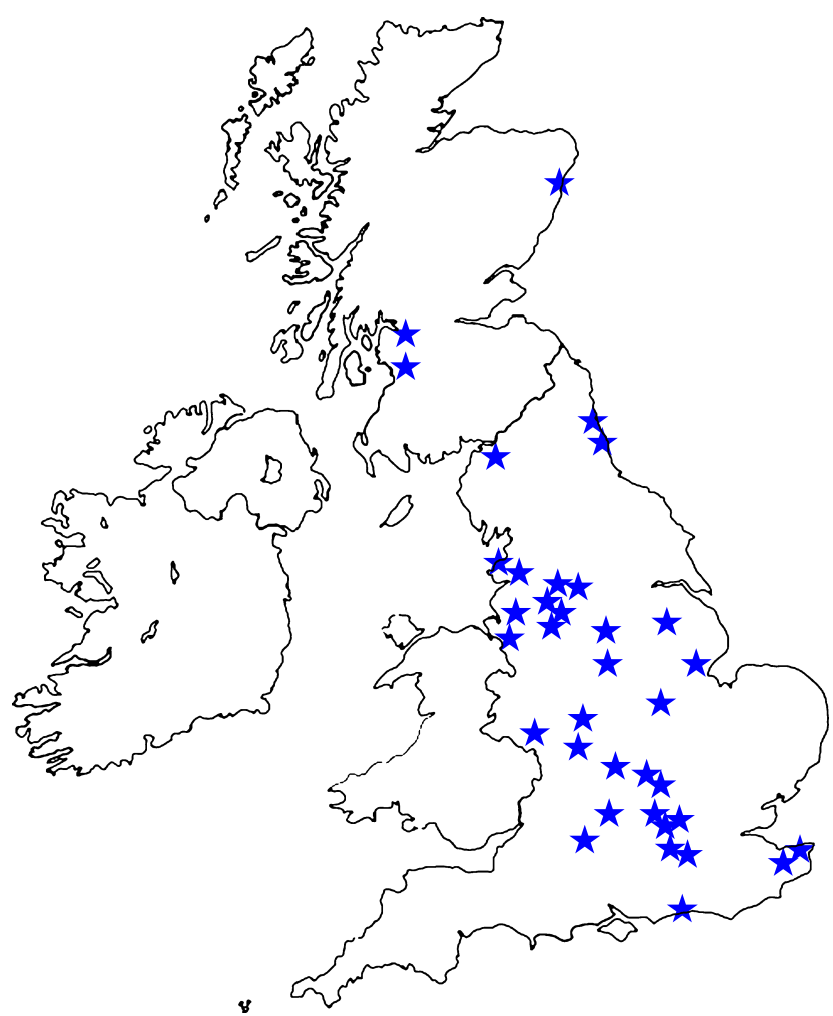

Fig. 6. Recruitment sites for the genetics of otitis media study April 2009 to December 2013. relative risk, amounting to around 3500 individual participants. The original plan was to recruit from eight regional hospitals in the Thames Valley area, but early recruitment was slow, and it transpired that unfortunately less than half of participants who agreed to participate actually returned their DNA. ${ }^{2}$ In the first 10 months of the study, an average of only nine families per month were successfully recruited, a trajectory that predicted that less than half of the recruitment target would be reached in the 3-4 years allocated to this study.

Inclusion onto the portfolio transformed the study (Fig. 5). Funds were provided for a full-time research nurse and a study administrator, and the study was advertised through the NIHR Portfolio website. Contact from research teams across the United Kingdom meant the study rapidly expanded to include 36 centres (Fig. 6). In the last 6 months of 2013, the study was recruiting an average of 45 families per month. The study was extended to recruit beyond the original sample size to enable analysis of genotypes of lower relative risk and has now recruited over 1600 families (around 6000 individuals) to the end of 2013, making this also the largest study in the world investigating the genetics of otitis media. That is all down to the support afforded through the CRN.

\section{Contact us}

This article has outlined how the NIHR is supporting clinical research in the NHS through infrastructure, clinical networks and training. The success stories are growing and the pace and quality of research within ENT is increasing. To take advantage of the support and delivery options available to researchers, we encourage ENT researchers to contact the CRN via their local 
network, through the main NIHR CRN website, or via the ENT specialty.

\section{How do I approach the CRN?}

- The NIHR CRN website http://www.crn.nihr.ac.uk/

- Your local network (LCRN) http://www.crn.nihr.ac. uk/about_us

- The ENT specialty http://www.crn.nihr.ac.uk/about_us/ear_nose_throat/

- The national ENT specialty lead a.schilder@ucl.ac.uk

- The national ENT specialty administrator m.simmonds@ucl.ac.uk

- Your ENT specialty local lead (Table 1)

\section{Keypoints}

- The NIHR CRN provides the infrastructure to support clinical research in the NHS

- ENT researchers can access CRN support by registering their studies onto the CRN Portfolio

- Two recently completed ENT clinical studies have attributed their success to the support they received from the NIHR CRN

\section{Conflict of interest}

None to declare.

\section{References}

1 Fokkens W.J., Lund V.J., Mullol J. et al. (2012) European position paper on rhinosinusitis and nasal polyps 2012. Rhinol. Suppl. 3, 1-298

2 Bhutta M.F., Hobson L., Lambie J. et al. (2013) Alternative recruitment strategies influence saliva sample return rates in community-based genetic association studies. Ann. Hum. Genet. 77, 244-250 